\title{
Exclusive vector meson photoproduction at Run 2 LHC energies: Color dipole predictions
}

\author{
Victor P. Goncalves* \\ High and Medium Energy Group, Instituto de Física e Matemática, UFPel \\ Caixa Postal 354, 96010-900, Pelotas, RS, Brazil. \\ E-mail:barroseufpel.edu.br
}

\section{V. T. Machado}

High Energy Physics Phenomenology Group, GFPAE IF-UFRGS

Caixa Postal 15051, CEP 91501-970, Porto Alegre, RS, Brazil.

E-mail:magno.machado@ufrgs.br

\section{B. D. Moreira}

High and Medium Energy Group, Instituto de Física e Matemática, UFPel

Caixa Postal 354, 96010-900, Pelotas, RS, Brazil.

E-mail: bduartesmegmail.com

\section{F. S. Navarra}

Instituto de Física, Universidade de São Paulo

C.P. 66318, 05315-970 São Paulo, SP, Brazil.

E-mail:navarraeif.usp.br

\section{G. Sampaio dos Santos}

High and Medium Energy Group, Instituto de Física e Matemática, UFPel

Caixa Postal 354, 96010-900, Pelotas, RS, Brazil.

E-mail: gss.fisica@gmail.com

\begin{abstract}
The theoretical uncertainty on the Color Dipole predictions for the $\rho$ and $J / \Psi$ photoproduction in nucleus-nucleus collisions at Run 2 LHC energies is investigated. The results for rapidity distributions and total cross sections are presented. Moreover, the predictions are compared with the recent preliminary data from ALICE collaboration for the $\rho$ photoproduction in $\mathrm{PbPb}$ collisions at central rapidities.
\end{abstract}

XXVI International Workshop on Deep-Inelastic Scattering and Related Subjects (DIS2018) 16-20 April 2018

Kobe, Japan

\footnotetext{
* Speaker.
} 


\section{Introduction}

The advent of the high-energy colliders has motivated the study of the hadron structure at high energies. In such scenario, a hadron becomes a dense system and the nonlinear effects inherent to the QCD dynamics may become visible. Such regime can be studied in $\gamma A$ interactions at the LHC, present in ultraperipheral collisions (UPC). The main advantage of using colliding hadrons and nuclear beams for studying photon induced interactions is the high photon-hadron center-ofmass energy and luminosities achieved at LHC. Consequently, studies of $\gamma A$ interactions at the LHC could provide valuable information on the QCD dynamics at high energies. During the last years, the LHC has provided data on vector meson photoproduction at Run 1 energies and in this year at Run 2 energies. The Run 2 at the LHC has already produced $P b P b$ collisions and more data in hadronic collisions are expected in the next years. These collisions are now performed at energies which are a factor 2 larger than those of Run 1. Here, our goal is to analyze the theoretical uncertainties on the predictions for the photoproduction of light and heavy vector mesons in $\mathrm{PbPb}$ collisions at the LHC using the color dipole approach.

\section{Formalism}

Let us start defining a UPC as a collision between two electric charges at impact parameters such that $b>R_{1}+R_{2}$, where $R_{i}$ is the radius of the charge $i$. In a UPC at high energies, it is well known that the hadrons act as a source of almost real photons. Consequently, the exclusive meson photoproduction in hadron-hadron collisions can be factorized in terms of the equivalent flux of photons of the hadron projectile and photon-target production cross section [1],

$$
\sigma\left(h_{1}+h_{2} \rightarrow h_{1} \otimes V \otimes h_{2}\right)=\int d \omega \frac{n_{h_{1}}(\omega)}{\omega} \sigma_{\gamma h_{2} \rightarrow V \otimes h_{2}}\left(W_{\gamma h_{2}}^{2}\right)+\int d \omega \frac{n_{h_{2}}(\omega)}{\omega} \sigma_{\gamma h_{1} \rightarrow V \otimes h_{1}}\left(W_{\gamma h_{1}}^{2}\right)
$$

where $n(\omega)$ is the equivalent photon spectrum and $\sigma_{\gamma h \rightarrow V \otimes h}\left(W_{\gamma h}^{2}\right)$ is the vector meson photoproduction cross section. Moreover, the photon energy $\omega$ and $W_{\gamma h}$, the c.m. energy of the $\gamma h$ system, are related by $W_{\gamma h}=\sqrt{4 \omega E}$, where $E=\sqrt{s} / 2$ and $\sqrt{s}$ is the hadron-hadron c.m. energy. The equivalent photon spectrum is well known and is fully computed in QED [1]. The cross section for the exclusive vector meson photoproduction can be expressed as follows

$$
\sigma(\gamma h \rightarrow V h)=\frac{1}{16 \pi} \int_{-\infty}^{0}\left|\mathscr{A}^{\gamma h \rightarrow V h}(x, \Delta)\right|^{2} d t .
$$

Here, the $\gamma h$ interaction will be described within the dipole frame, where the probing projectile fluctuates into a quark-antiquark pair with transverse separation $\boldsymbol{r}$ (and momentum fraction z), which scatters off the hadron target and then forms a vector meson at the final state. If the lifetime of the dipole is much larger than the interaction time, a condition which is satisfied in high energy collisions, the scattering amplitude $\mathscr{A}^{\gamma h \rightarrow V h}(x, \Delta)$ can be written as [2],

$$
\mathscr{A}^{\gamma h \rightarrow V h}(x, \Delta)=i \int d z d^{2} r d^{2} b_{h}\left[\Psi^{V *}(r, z) \Psi(r, z)\right]_{T} e^{-i\left[b_{h}-(1-z) r\right] \cdot \Delta} 2 \mathscr{N}_{h}\left(x, r, b_{h}\right),
$$

where $\Psi^{V *}(r, z)$ and $\Psi(r, z)$ are the wave functions of the photon and of the vector meson, respectively. The overlap function $\left[\Psi^{V *}(r, z) \Psi(r, z)\right]_{T}$ describes the fluctuation of the photon with 
transverse polarization into a color dipole and the subsequent formation of the vector meson. All the information about the strong interactions in the process is encoded in the forward dipole-hadron scattering amplitude $\mathscr{N}_{h}\left(x, r, b_{h}\right)$. The variable $b_{h}$ stands for the impact parameter, the separation between the dipole center and the target center, $x=M_{V}^{2} / W^{2}$ is the Bjorken variable and $\Delta$ is related to the momentum transfer squared by $\Delta=\sqrt{-t}$. In the numerical evaluations in next section, we have considered the Boosted Gaussian (BG) [3] and the Light-Cone Gaussian (LCG) [4] wavefunctions and the phenomenological models for dipole scattering amplitude: IIM [5], bCGC [6, 2] and IP-SAT [7] models, which encode the main properties of the saturation approaches. Such models are based on the Color Glass Condensate formalism and describe the HERA data taking into account the nonlinear effects in the QCD dynamics. We have considered the set of parameters for the IIM and bCGC parameterizations from Ref.[9]. The IP-SAT model uses an eikonalized form for $\mathscr{N}_{p}$ that depends on a gluon distribution evolved via DGLAP equation. In this work we calculated the IP-SAT predictions using a FORTRAN library provided by the authors of Ref.[10], which includes an updated analysis of combined HERA data. In order to estimate $\gamma A$ process, we need to adapt the phenomenological models described above to the nuclear case. We will assume the model proposed in Ref.[11] that contains the impact parameter dependence, where the dipole-nucleus scattering amplitude is written as

$$
\begin{aligned}
\mathscr{N}_{A} & =1-\exp \left[-\frac{1}{2} \sigma_{\text {dip }}\left(x, r^{2}\right) T_{A}\left(b_{A}\right)\right], \\
\sigma_{d i p}\left(x, r^{2}\right) & =2 \int d^{2} b_{p} \mathscr{N}_{p}\left(x, r, b_{p}\right),
\end{aligned}
$$

where $T_{A}\left(b_{A}\right)$ is the nuclear thickness. The above equation sums up all the multiple elastic rescattering diagrams of the $q \bar{q}$ pair and is justified for large coherence length, where the transverse separation $r$ of partons in the multiparton Fock state of the photon becomes a conserved quantity, i.e. the size of the pair $r$ becomes eigenvalue of the scattering matrix.

\section{Results}

Before presenting the predictions for the vector meson production we will compute $\mathscr{N}_{A}$ considering the different models for the dipole-proton scattering amplitude discussed previously. In Fig. 1 we show the results for the nuclear scattering amplitude take into account the IIM, bCGC and IP-SAT models considering different values of the impact parameter $b_{A}$. As expected, $\mathscr{N}_{A}$ saturates faster for central collisions than for large impact parameters. Moreover, the deviation between the predictions is small. This is directly associated with the model for $\mathscr{N}_{A}$, given by Eq.(2.4), which is the same in all three cases. The future experimental data on vector meson photoproduction in $\mathrm{PbPb}$ collisions will be useful to test this model of $\mathscr{N}_{A}$.

In Fig.2 we present our predictions [12] (which are a complement the predictions presented in Ref.[13]) for the exclusive photoproduction of $\rho$ and $J / \Psi$ in $P b P b$ collisions at $\sqrt{s}=5.02$ $\mathrm{TeV}$. As we can see the difference between the predictions is not large, as expected from Fig.1. In particular, at central rapidities the bCGC and IP-SAT parameterizations deliver quite similar results. Moreover, in Fig. 2 we compare our results with the recent preliminary data on $\rho$ photoproduction at central rapidity released by ALICE Collaboration [14]. We can verify that the color dipole 

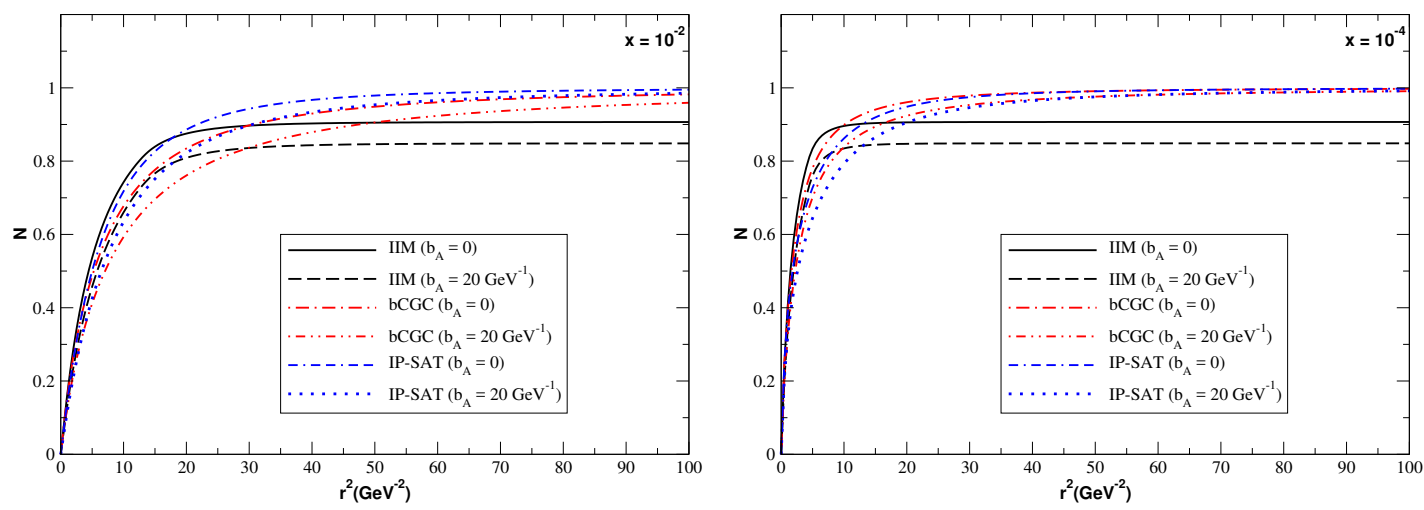

Figure 1: Dipole-nucleus scatering amplitude as a function of $r^{2}$ for fixed values of $b$ and different values of $x(A=P b)$.

\begin{tabular}{|c|c|c|}
\hline & $\rho$ & $J / \Psi$ \\
\hline $\mathrm{PbPb}(\sqrt{s}=5.02 \mathrm{TeV})$ & $5.26-7.04 \mathrm{~b}$ & $18.24-24.47 \mathrm{mb}$ \\
\hline
\end{tabular}

Table 1: The total cross sections of the photoproduction of $\rho$ and $J / \Psi$ in $P b P b$ collisions at the Run 2 LHC energies.

predictions overestimate the data. This result is an indication that other effects, not included in our analysis, should be included at least for this final state. Some possibilities are the inclusion of shadowing corrections [8] or absorption corrections [15]. Another possible conclusion is that the treatment of the dipole-nucleus interaction, described here by the model presented in Eq.(2.4), should be improved.
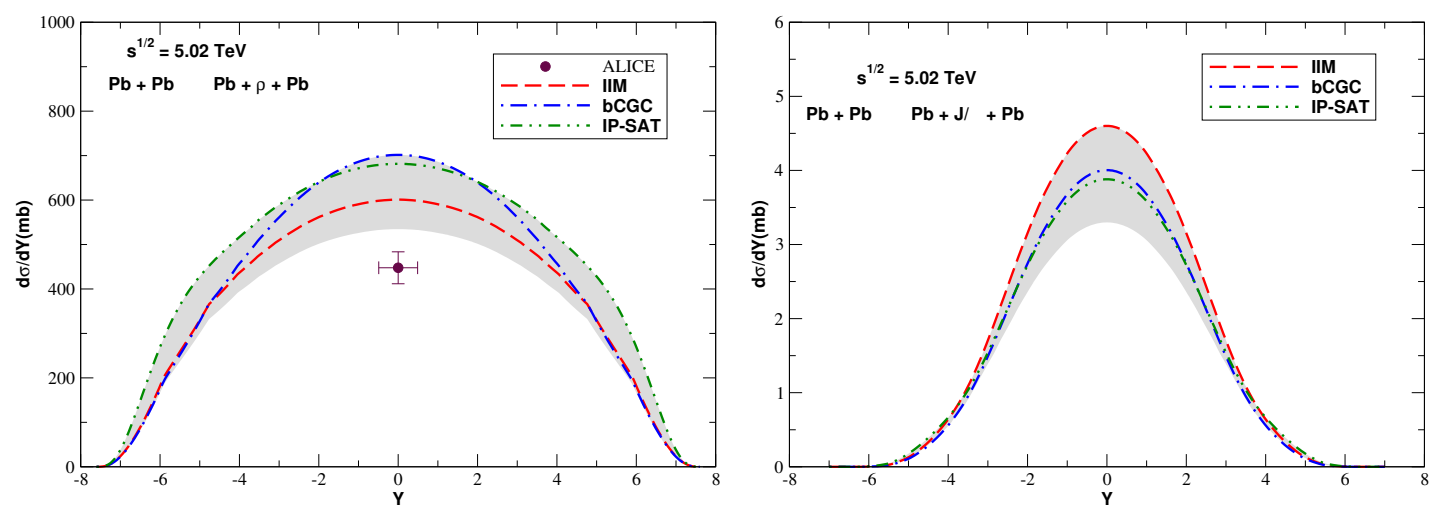

Figure 2: Rapidity distributions for the photoproduction of $\rho$ and $J / \Psi$ in $P b P b$ collisions at $\sqrt{s}=5.02 \mathrm{TeV}$.

Finally, in order to estimate the dependence of our results on the models used (for the dipole scattering amplitude and for the vector meson wavefunctions), we present in Tab.1 the lower and upper bounds of our predictions for the total cross sections. As expected from the analysis of the rapidity distributions, the largest uncertainties are presented in the $\rho$ production. Additionally, the cross sections for $\mathrm{PbPb}$ collisions decrease with the mass of the vector meson. 


\section{Summary}

We have presented predictions for the exclusive $\rho$ and $J / \Psi$ photoproduction in $P b P b$ collisions. Our results demonstrated that cross section for the light meson production is larger than the heavy meson one. Moreover, the light meson production suffers from the largest theoretical uncertainties on the predictions. The comparison with the preliminary experimental data on the $\rho$ production in $\mathrm{PbPb}$ collisions indicated that the current color dipole description overestimate the data. This can be interpreted as an indication that a more careful treatment of the dipole-nucleus interaction and/or another effects as shadowing and absorptive corrections should be incorporated to the formalism.

\section{Acknowledgments}

This work was partially financed by the Brazilian funding agencies CAPES, CNPq, FAPERGS and FAPESP.

\section{References}

[1] V. P. Goncalves and M. V. T. Machado, Mod. Phys. Lett. A 19, 2525 (2004).

[2] H. Kowalski, L. Motyka and G. Watt, Phys. Rev. D 74, 074016 (2006).

[3] J. Nemchik, N. N. Nikolaev, E. Predazzi and B. G. Zakharov, Z. Phys. C 75, 71 (1997) 71.

[4] H.G. Dosch, T. Gousset, G. Kulzinger and H.J. Pirner, Phys. Rev. D55, 2602 (1997).

[5] E. Iancu, K. Itakura and S. Munier, Phys. Lett. B 590, 199 (2004).

[6] G. Watt and H. Kowalski, Phys. Rev. D 78, 014016 (2008).

[7] J. Bartels, K. J. Golec-Biernat and H. Kowalski, Phys. Rev. D 66, 014001 (2002).

[8] G. Sampaio dos Santos and M.V.T. Machado, Phys. Rev. C 89, 025201 (2014); Phys. Rev. C 91, 025203 (2015).

[9] A. H. Rezaeian and I. Schmidt, Phys. Rev. D 88, 074016 (2013).

[10] A. H. Rezaeian, M. Siddikov, M. Van de Klundert and R. Venugopalan, Phys. Rev. D 87, 034002 (2013).

[11] N. Armesto, Eur. Phys. J. C 26, 35 (2002).

[12] V. P. Goncalves et al., Phys. Rev. D 96, no. 9, 094027 (2017).

[13] V. P. Goncalves, B. D. Moreira and F. S. Navarra, Phys. Rev. D 95, no. 5, 054011 (2017).

[14] E. L. Kryshen [ALICE Collaboration], Nucl. Phys. A 967, 273 (2017)

[15] S. P. Jones, A. D. Martin, M. G. Ryskin and T. Teubner, JHEP 1311, 085 (2013). 